\title{
Radioligand therapy of metastatic castration-resistant prostate cancer: current approaches
}

\author{
Zool Hilmi Awang, Markus Essler and Hojjat Ahmadzadehfar*
}

\begin{abstract}
Prostate Cancer is the forth most common type of cancer. Prostate-specific membrane antigen (PSMA) is anchored in the cell membrane of prostate epithelial cells. PSMA is highly expressed on prostate epithelial cells and strongly up-regulated in prostate cancer. Therefore it is an appropriate target for diagnostic and therapy of prostate cancer and its metastases. This article discusses several articles on radionuclide treatments in prostate cancer and the results on PSMA therapy with either beta or alpha emitters as a salvage therapy.
\end{abstract}

Keywords: PSMA, Prostate cancer, Radioligand therapy, Metastatic disease

\section{Background}

Prostate cancer is the fourth most common type of cancer affecting the European male population (excluding non-melanoma skin cancer) [1]. Currently, one in every six males are at risk of being affected by prostate cancer, and the risk of death because of metastatic prostate cancer is one in every 30 [2]. Castration-resistant prostate cancer (CRPC) is defined by disease progression despite castrated levels of testosterone, and may present as either a continuous rise in serum prostate-specific antigen (PSA) levels, the progression of pre-existing disease, and/or the appearance of new metastases [3].

In patients who fail the initial therapy with curative intent (i.e. radical prostatectomy, external beam radiotherapy [EBRT], brachytherapy) treatment options include androgen-deprivation therapy (ADT) along with chemotherapy in the case of disease progression [4]. A combination of ADT with docetaxel in hormone-sensitive patients improves median overall survival (OS) by 13.6 months compared to ADT alone [5, 6]. In CRPC patients, a more recent approach using abiraterone and enzalutamide prolongs median survival by up to 3.9 and 4.8 months, respectively $[7,8]$. Chemotherapy treatment with docetaxel and cabazitaxel is often associated with side effects but prolongs OS for a few

* Correspondence: hojjat.ahmadzadehfar@ukbonn.de

Department of Nuclear Medicine, University Hospital Bonn,

Sigmund-Freud-Str. 25, 53127 Bonn, Germany months $[4,9,10]$. Furthermore, treatment for diffused or painful bone metastases using radium-223-chloride $\left({ }^{223} \mathrm{Ra}\right)$, which targets only the osteoblastic lesions and does not treat the nodal and visceral metastases, improves median OS by 3.6 months [11].

Positron emission tomography/computed tomography (PET/CT) using Gallium-68-labelled (Ga-68) ligands that target the prostate-specific membrane antigen (PSMA) is a sensitive and specific diagnostic method that is dedicated to poorly differentiated prostate cancer. Eiber et al. [12] reported that sensitivity increased to $100 \%$ with an increasing PSA velocity of $5 \mathrm{ng} / \mathrm{ml} /$ year or greater and with a Gleason score of eight or more. The use of PET/CT to target PSMA with Ga-68 for diagnostic and radioligand therapy (RLT) with Lutetium-177 offers a new theranostics approach using the same ligand for diagnostics and therapy [13]. Since 2013, an increasing number of centres worldwide has begun employing radioligand therapy (RLT) using ${ }^{177}$ Lu-PSMA [14-17].

The aim of this review is to discuss the current trend of using ${ }^{177}$ Lu-PSMA therapy, including dosimetry, side effects, treatment efficacy and survival rates, while referring to the literature and examining the prospects for prostate cancer therapy with targeted alpha therapy.

\section{Indications for RLT}

Metastatic castration-resistant prostate cancer (mCRPC) patients can undergo treatment with taxane-based

(c) The Author(s). 2018 Open Access This article is distributed under the terms of the Creative Commons Attribution 4.0 International License (http://creativecommons.org/licenses/by/4.0/), which permits unrestricted use, distribution, and reproduction in any medium, provided you give appropriate credit to the original author(s) and the source, provide a link to the Creative Commons license, and indicate if changes were made. The Creative Commons Public Domain Dedication waiver (http://creativecommons.org/publicdomain/zero/1.0/) applies to the data made available in this article, unless otherwise stated. 
therapies (docetaxel and cabazitaxel) and with second line hormonal therapies (including enzalutamide and abiraterone). Both these therapies moderately improve patient survival time, but they are only temporarily effective and patients can develop resistance [18, 19]. Hence, more specific targeted therapies have to be developed for eliminating the prostate cancer visceral and bony lesions. Studies have shown that PSMA is overexpressed in around $90-100 \%$ of local prostate cancer lesions, along with many bony lesions and lymph node metastases. Furthermore, many studies have shown that the PSMA expression levels increase in the case of metastatic, high-grade and castration-resistant prostate cancer [20-22] (Fig. 1). The current essential inclusion criteria, as stated in the 2016 consensus recommendations of the German Society of Nuclear Medicine [23], cover:

1) histologically detected prostate carcinomas;

2) non-resectable metastases;

3) tumour progression under guidelines therapy;

4) detected PSMA expression of the tumour;

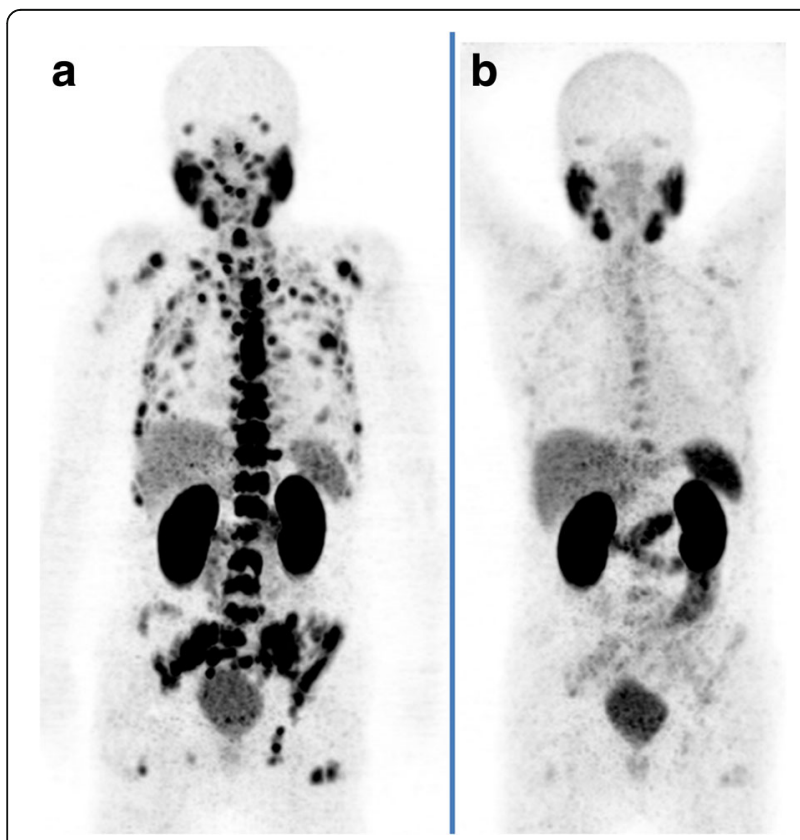

Fig. 1 A 83-year-old patient with castration-resistant prostate cancer (Gleason-Score:9) and an increasing prostate-specific antigen (PSA) level. He had a history of prostatectomy and radiation therapy of prostate bed. The 68 Ga-PSMA PET scan showed a diffuse bone and bone marrow involvement (a). The PSA and ALP levels prior to the first cycle of Lu-PSMA therapy were $261 \mathrm{ng} / \mathrm{ml}$ and $659 \mathrm{U} /$, respectively. The patient received 2 cycles of Lu-PSMA and the PSA level decreased continuously during cycles from 261 to $9.0 \mathrm{ng} / \mathrm{ml}$ (8 weeks after the second cycle). The ALP showed also a decreasing value from 659 to $81 \mathrm{U} / \mathrm{l}$ (8 weeks after the second cycle). The PSMA-PET (b) 8 weeks after the second cycle showed a significant response with significant regression of PSMA
5) reasonable haematological function (leukocyte count $>2.0 \times 10^{9} / \mathrm{L}$, thrombocyte $>75 \times 10^{9} / \mathrm{L}$;

6) normal or slightly decreased renal function (creatinine $<2 x$ the upper standard limit);

7) sufficient liver function (aspartate aminotransferase $[\mathrm{AST}]$ or alanine aminotransferase $[\mathrm{ALT}]<5 \mathrm{x}$ the upper standard limit); and

8) a six-week interval with myelosuppressive therapy.

\section{Activity level}

The standard administered activity of ${ }^{177}$ Lu-PSMA has varied across the literature as institutions undertake safety and toxicity trials [16]. Single injected doses have ranged from 3 to $9.3 \mathrm{GBq}$, with up to nine injections given to patients, generally at a minimum of six-week intervals $[14,24-31]$.

\section{Response rate}

Up to $80 \%$ of patients with mCRPC will have a treatment response to ${ }^{177} \mathrm{Lu}-\mathrm{PSMA}$ shown by any PSA decline [14, 24-30, 32-34] (Table 1).

Studies using ${ }^{177} \mathrm{Lu}$-PSMA-617 and ${ }^{177} \mathrm{Lu}$-PSMA-I\&T have observed a reduction in PSA levels by $50 \%$ or more in $32-60 \%$ of patients. Moreover, $47 \%$ of patients have experienced a stable disease [14, 24-30, 32-34]. In 2016, a group from Heidelberg, Germany, started the first human treatments with ${ }^{255}$ Ac-PSMA-617 in two patients with red marrow infiltration and resistance to other therapies, and these patients showed complete responses to the therapy [35, 36]. A study by Zechmann et al., using a ${ }^{131}$ I-labelled PSMA ligand, showed a $50 \%$ or greater decline in PSA in more than $60 \%$ of patients [37]. This finding was in line with a recent study by Afshar-Oromieh et al. who studied 36 patients who had received PSMARLT with ${ }^{131}$ I-IMP-1095, and found the best therapeutic effect was achieved by the first therapy, which showed that PSA declined by more than $50 \%$ in $70.6 \%$ of the patients. The second and third therapies from their study showed reduced effectiveness [38].

\section{Predictors of the response}

Ferdinandus et al. evaluated the prognostic value of various pre-therapeutic parameters on therapy response, based on changes in PSA after the first cycle of RLT. Their multivariate analysis of these parameters, which considered any decrease in PSA after 2 months, showed that patients with a high platelet count or a regular need for analgesics had a significantly worse response to the first RLT cycle. When a PSA decline of $\geq 50 \%$ was considered, patients with a regular need for analgesics showed a worse response in the multivariate analysis; however, other pre-therapeutic parameters had no impact on the response to RLT. In this study, the standard uptake value maximum of ${ }^{68} \mathrm{Ga}$-PSMA-11 was not a 


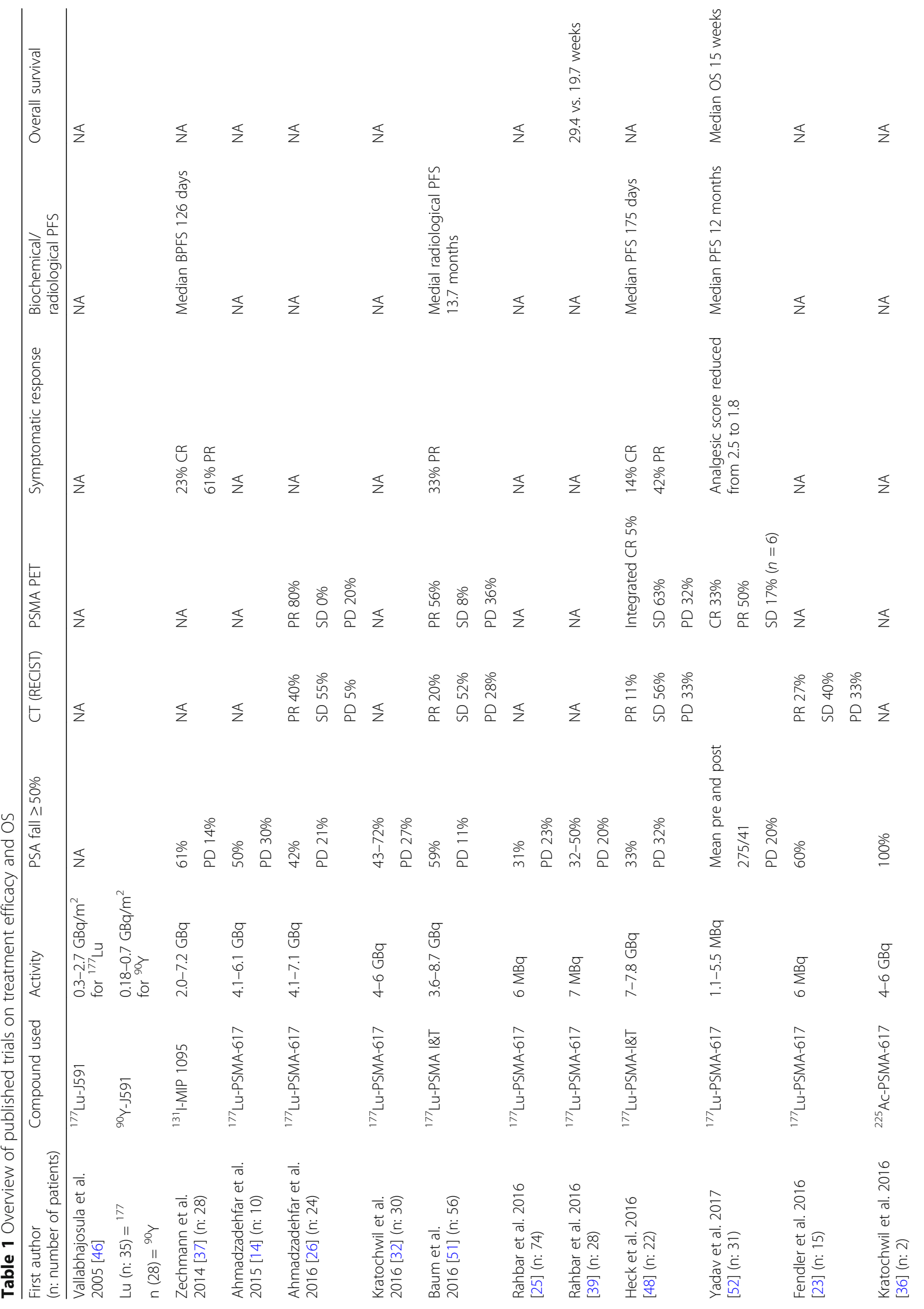




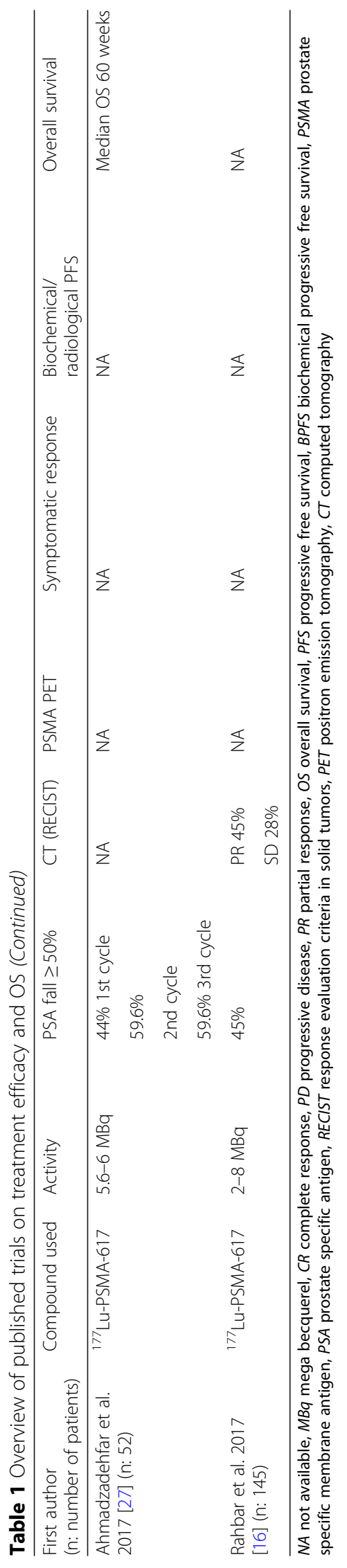


significant predictor of the response to RLT. One explanation for this could be that more aggressive tumours may express higher PSMA levels. However, despite the better uptake of ${ }^{177}$ Lu-PSMA-617 due to the rapid growth of metastases, the response rate did not correlate with the uptake, which could be due to different washout times of ${ }^{177} \mathrm{Lu}-\mathrm{PSMA}-617$ in the respective metastases [29].

\section{Survival}

Rahbar et al. reported a potential survival benefit of ${ }^{177} \mathrm{Lu}$-PSMA, where they matched the patient population ( $n=28$ ) to a historical cohort of 20 patients receiving the best supportive care (BSC) to examine potential survival benefits. Apart from the more heavily pre-treated patients and the more visceral metastases in the ${ }^{177}$ Lu-PSMA group, the groups were comparable. This finding highlights that the estimated median survival period was 29.4 weeks, being significantly longer than the survival time in the historical control group at 19.7 weeks [39].

In a study by Ahmadzadehfar et al. with 52 patients who underwent a total of 190 cycles of RLT, $80.8 \%$ of patients showed a decline in PSA levels 2 months after the first cycle, with $44.2 \%$ showing a PSA decline of $\geq 50 \%$. The median OS was 60 weeks in all patients. The median OS was significantly longer for patients who showed a PSA decline after the first cycle compared to patients without a PSA decline (68 versus 33 weeks respectively) [27]. In another study from the same group, 100 patients who received a total of 347 cycles of ${ }^{177} \mathrm{Lu}$-PSMA (median three cycles) were analysed. All patients had a history of therapy with abiraterone or enzalutamide, or both. In total, $70 \%$ of the patients had at least one line of chemotherapy and $36 \%$ had a history of radionuclide therapy with ${ }^{223} \mathrm{Ra}$. Sixty-nine patients showed a decline in PSA 2 months after the first cycle, and 38 of these patients showed a PSA decline of $\geq 50 \%$. The median OS was 60 weeks. In the multivariate analysis, the median OS was significantly longer in those patients without hepatic involvement, with high levels of albumin and haemoglobin $(\mathrm{Hb})$, and with low levels of AST. Moreover, in the univariate analysis, a PSA decline after the first RLT, as well as any decline $>50 \%$, were significant predictors of a longer OS. A decline in PSA levels of more than $14 \%$ was the most important response parameter with regard to OS [40]. In a bicentric study, 104 patients were treated with 351 cycles of ${ }^{177} \mathrm{Lu}-\mathrm{PSMA}-617$. All of them had a history of therapy with at least one line of chemotherapy as well as either abiraterone or enzalutamide. Thus, in this study, the patients received all recommended guideline therapies. A PSA decline occurred in 70 (67\%) patients, with a PSA decline $\geq 50 \%$ in 34 (33\%) patients after the first cycle. The median OS was 56.0 weeks (95\% CI: 50.5-61.5). Any initial PSA decline, an initial alkaline phosphatase $(\mathrm{ALP})<220 \mathrm{U} / \mathrm{L}$ and a cumulative injected activity of $\geq 18.8 \mathrm{GBq}$ were associated with a longer survival. A step-by-step analysis revealed a PSA decline of $\geq 20.9 \%$ as the most noticeable cut-off prognosticating longer survival, which remained an independent prognosticator of improved OS in the multivariate analysis [41]. These studies have shown that responders to PSMA therapies live longer than non-responders, and a PSA response should not necessarily be defined as a PSA decline of $>50 \%$. Interestingly, prior therapies, such as chemotherapy, had no impact on OS.

\section{Dosimetry}

The distribution of small molecules of PSMA ligands in tissue is quick and, over time, the uptake in prostate cancer tissue increases, whereas the uptake in healthy tissue declines [42]. In normal healthy tissue, salivary glands have the highest PSMA binding, followed by normal kidney tissue.

Kabasakal et al. [15] reported their dosimetry results with ${ }^{177} \mathrm{Lu}$-PSMA-617 and showed the highest radiation estimated doses in parotid glands and kidneys. Calculated radiation-absorbed doses per megabecquerel were $1.17 \pm 0.31 \mathrm{mGy}$ for parotid glands and $0.88 \pm 0.40 \mathrm{mGy}$ for kidneys. The radiation dose given to bone marrow was significantly lower than those of kidney and parotid glands $(p<0.05)$. The calculated radiation dose to bone marrow was $0.03 \pm 0.01 \mathrm{mGy} / \mathrm{MBq}$.

These results were reproduced by Delker et al. [43] who reported their dosimetry results with ${ }^{177}$ Lu-PSMA-617 and calculated a mean absorbed dose to bone marrow, kidneys, liver, spleen, and salivary glands of $0.012 \mathrm{~Gy} / \mathrm{GBq}$, $0.6 \mathrm{~Gy} / \mathrm{GBq}, 0.1 \mathrm{~Gy} / \mathrm{GBq}, 0.1 \mathrm{~Gy} / \mathrm{GBq}$ and $1.4 \mathrm{~Gy} / \mathrm{GBq}$ respectively.

There are several profound fears with regards to the damage caused to salivary glands. Based on external beam radiotherapy (EBRT) data, irreversible damage to salivary glands occurs after the administration of 30-40 Gy. With a mean absorbed dose of $1.4 \mathrm{~Gy} / \mathrm{GBq}$ of ${ }^{177} \mathrm{Lu}$-PSMA-617, and the absence of permanent xerostomia or hypogeusia in the initial treatment studies, the salivary glands do not appear to be a dose-limiting organ [43].

The second fear concerns the absorbed dose to kidney tissues $[15,43]$, where, based on the EBRT data, a dose of 23 Gy may result in permanent damage. The mean absorbed kidney dose of ${ }^{177} \mathrm{Lu}$-PSMA is $0.53-0.8 \mathrm{~Gy} / \mathrm{GBq}$, quite like the absorbed kidney dose mentioned in the published data on ${ }^{177} \mathrm{Lu}$-DOTATATE $(0.64 \pm 0.16 \mathrm{~Gy} / \mathrm{GBq})$ [44].

In a study with 135 patients undergoing diagnostics using ${ }^{68}$ Ga-PSMA PET-CT, Gaertner et al. [45] compared three groups of patients with low, moderate, and high tumour loads according to their tumour volume. Their results indicate that patients with high tumour loads may receive less toxicity in their nontarget organs. 
As a result of previous findings, the safety and efficacy of targeted radionuclide therapies can be improved using patient-specific dosimetry, which may help to guide successful tumour dosing and act as an early indicator of organ toxicity.

\section{Toxicity}

\section{Myelosuppression}

Myelotoxicity is a classic non-stochastic (deterministic) effect. This effect is characterised by a sigmoidal, doseresponse relationship [46].

A report from a German multicentre study [16] showed that grade 3-4 hematologic adverse events occurred in 18 of 145 patients (12\%). Furthermore, one (0.7\%) patient experienced severe leukopenia, 11 (8\%) patients experienced anaemia, two (2\%) patients experienced thrombocytopenia, and four (3\%) patients had a combination of these conditions.

Ahmadzadehfar et al. [28] showed in a retrospective analysis of 49 patients, who had undergone three cycles of RLT with at least 2 months of follow up after the last cycle, that there was no CTC $4^{\circ}$ haematotoxicity in the entire study population. Relevant anaemia, thrombocytopenia and leukopenia (CTC $3^{\circ}$ ) occurred during the observation period after the third cycle in four (8.2\%), three (6.1\%) and zero patients, respectively. The patients were divided into two groups with regard to their history of therapy with ${ }^{223} \mathrm{Ra}$. Group 1 included 20 patients who had received therapy with ${ }^{223} \mathrm{Ra}$ (median six cycles) prior to ${ }^{177}$ Lu-PSMA-617 therapy. Group 2, which was the control group regarding haematotoxicity, was comprised of 29 patients without any history of a bone-targeted radionuclide therapy. There was no significant difference between the groups regarding relevant haematotoxicity. Thus, according to the results of this study, performing repeated cycles of ${ }^{177} \mathrm{Lu}$-PSMA-617 after ${ }^{223}$ Ra seems to be safe, with a very small probability of haematotoxicity [28].

\section{Renal toxicity}

Due to the physiological expression of PSMA in kidneys, many researchers have been concerned about probable radiation toxicity to the kidneys. Along with glomerular filtration rate (GFR) and creatinine levels, renal scintigraphy should be performed with Tc-MAG3 before therapy for overruling any significant obstructive disease. Any relevant obstructive disease must be treated in order to decrease the radiation dose to the diseased kidneys. Recently, Yordanova et al. [47] reported on 55 patients treated with ${ }^{177} \mathrm{Lu}-\mathrm{PSMA}-617$, where 14 (25\%) showed a (sub-)acute toxicity of CTC $1^{\circ}$ and only one patient had a CTC $2^{\circ}$ according to the creatinine value. No grade 3-4 acute loss of renal function was detected, and this was in line with the German multicentre study [16]. A decreased GFR was observed in 16 patients
(29\%) where four had CTC $1^{\circ}$ and 12 had CTC $2^{\circ}$ toxicity [47]. It has been suggested that conditions that may affect renal function and increase the radiation absorbed dose to the kidneys occur in patients who are older-age men, who have had prior chemotherapy and who have accompanying diseases, such as hypertension [47] (Table 2).

\section{Salivary glands}

Although the salivary glands contain highly differentiated cells and their proliferation rate is slow, they are exceptionally radiosensitive organs. Due to the high binding of PSMA ligands, damage to the salivary glands and the development of xerostomia is a frequent side effect of radiation therapy which decreases the patient's quality of life. In the studies by Ahmadzadehfar et al. [26-28, 40], Heck et al. [48], and Rahbar et al. [14, 25, 41], patients received an ice pack collar for $30 \mathrm{~min}$ before and for up to $4 \mathrm{~h}$ after the administration of ${ }^{177} \mathrm{Lu}-\mathrm{PSMA}-617$ to induce vasoconstriction and reduce PSMA binding to the salivary glands. Transient xerostomia or hypogeusia occurred in $4-37 \%$ of patients with or without an ice pack collar $[16,26,48]$.

\section{Toxicity of ${ }^{225}$ AC-PSMA}

As stated earlier, ${ }^{225}$ Ac-PSMA therapy can be used in the patients who are unresponsive to ${ }^{177} \mathrm{Lu}$-PSMA therapy or show pronounced bone marrow infiltration. ${ }^{225}$ Ac-PSMA radiation consists of short-ranged alpha particles which kill tumour cells but spare the bone marrow cells. In a recent study, many patients reported the success of their ${ }^{225} \mathrm{Ac}$ PSMA therapy [36]. Also, in the case of the preclinical models of neuroendocrine tumours, ${ }^{225}$ Ac-DOTATOC was very effective in controlling the tumours, thus indicating that ${ }^{225}$ Ac-PSMA therapy was very effective [49]. In one recent study, researchers treated 40 patients using ${ }^{225}$ Ac-PSMA therapy and noted that four patients had to discontinue their treatment because of xerostomia. Twenty four patients (63\%) showed more than a 50\% decrease in their PSA levels while 33 (87\%) patients showed some PSA response. The median duration for tumour control after the last-line of ${ }^{225}$ Ac-PSMA-617 therapy was 9 months, while five patients endured a response for $\geq 2$ years [36, 50]. None of the patients showed any hematologic toxicity, and xerostomia was the only clinical side effect observed [36].

\section{Conclusions}

${ }^{177} \mathrm{Lu}$ - and ${ }^{225}$ Ac-based PSMA-targeted therapies appear to be promising and effective treatments for advanced prostate cancer. The new types of diagnostic tracers show a high sensitivity and specificity in the imaging of prostate cancers, even in patients with very low PSA levels, which has helped in the diagnostics, especially for staging and follow up during RLT. Therefore, prospective 


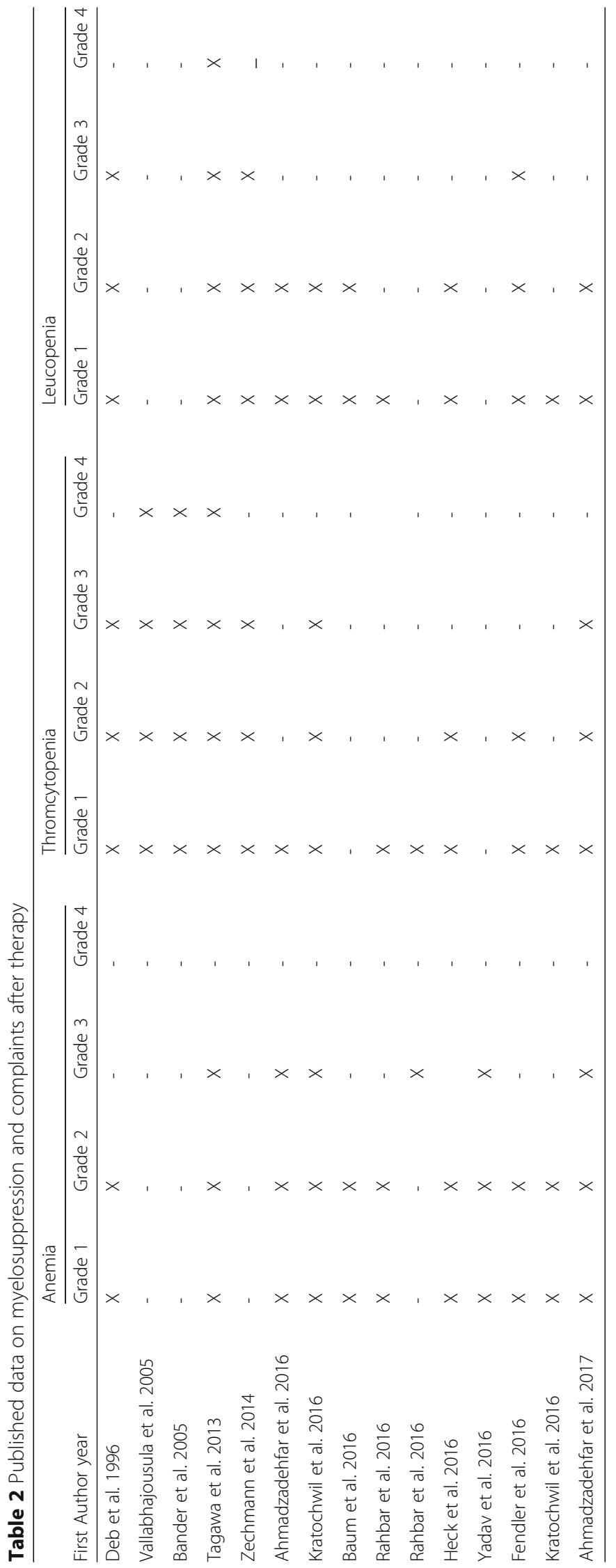


randomised trials are required to determine the impact of ${ }^{177} \mathrm{Lu}$-PSMA on survival, toxicities, dosimetry, and to rigorously assess the clinical benefits compared to other treatments for prostate cancer, including chemotherapy, EBRT, and androgen blockade. We suggest that a new type of nuclear medicine specialist should be established to perform and further develop radionuclide therapy: theranostics specialists should be trained in the fields of radionuclide treatment, radionuclide imaging, oncology, and radiation therapy to cover all aspects of these complex therapies and to ensure that this new treatment option is accepted worldwide.

\section{Abbreviations \\ ALT: Alanine aminotransferase; AST: Aspartate aminotransferase; CRPC: Castration-resistant prostate cancer; CTC: Common toxicity criteria; EBRT: External beam radiotherapy; Hb: Haemoglobin; MBq: Megabecquerel; OS: Overall survival; PET/CT: Positron emission tomography/computed tomography; PSA: Prostate-specific antigen; PSMA: Prostate-specific membrane antigen; RLT: Radioligand therapy; SUV: Standard uptake value}

\section{Acknowledgments}

We are grateful to the nursing staff of the treatment ward in our department. We give special thanks to our study nurse, Mrs. Ulrike Kuhn-Seifer (Department of Nuclear Medicine Bonn) and to the patients for their willingness to undergo an investigational treatment and consent to the publications of the results.

\section{Funding}

This research did not receive any specific grant from any funding agency in the public, commercial or not-for-profit sector.

\section{Authors' contributions}

Conception and design: HA, ME. Research: HA, ZHA. Manuscript writing: ZHA, HA, ME. All authors read and approved the final manuscript.

\section{Ethics approval and consent to participate}

All procedures performed in studies involving human participants were in accordance with the ethical standards of the institutional and/or national research committee and with the 1964 Helsinki Declaration and its later amendments or comparable ethical standards. This article does not contain any studies with animals performed by any of the authors.

\section{Consent for publication}

The consent form was signed by the patient presented in Fig. 1.

\section{Competing interests}

The authors declare that they have no competing interests.

\section{Publisher's Note}

Springer Nature remains neutral with regard to jurisdictional claims in published maps and institutional affiliations.

Received: 7 December 2017 Accepted: 27 April 2018

Published online: 23 May 2018

\section{References}

1. Ferlay J, Steliarova-Foucher E, Lortet-Tieulent J, et al. Cancer incidence and mortality patterns in Europe: estimates for 40 countries in 2012. Eur J Cancer. 2013;49:1374-403.

2. Nelson WG, De Marzo AM, Isaacs WB. Prostate cancer. N Engl J Med. 2003;349:366-81.

3. Saad F, Chi KN, Finelli A, et al. The 2015 CUA-CUOG guidelines for the management of castration-resistant prostate cancer (CRPC). Can Urol Assoc J. 2015;9:90-6.

4. Cornford P, Bellmunt J, Bolla M, et al. EAU-ESTRO-SIOG guidelines on prostate Cancer. Part II: treatment of relapsing, metastatic, and castration-resistant prostate Cancer. Eur Urol. 2017;71:630-42.
5. Sweeney CJ, Chen YH, Carducci M, et al. Chemohormonal therapy in metastatic hormone-sensitive prostate cancer. N Engl J Med. 2015;373:737-46.

6. James ND, Sydes MR, Clarke NW, et al. Addition of docetaxel, zoledronic acid, or both to first-line long-term hormone therapy in prostate cancer (STAMPEDE): survival results from an adaptive, multiarm, multistage, platform randomised controlled trial. Lancet. 2016;387:1163-77.

7. Chi KN, Kheoh T, Ryan CJ, et al. A prognostic index model for predicting overall survival in patients with metastatic castration-resistant prostate cancer treated with abiraterone acetate after docetaxel. Ann Oncol. 2016;27:454-60.

8. Scher HI, Fizazi K, Saad F, et al. Increased survival with enzalutamide in prostate cancer after chemotherapy. N Engl J Med. 2012;367:1187-97.

9. de Bono JS, Oudard S, Ozguroglu M, et al. Prednisone plus cabazitaxel or mitoxantrone for metastatic castration-resistant prostate cancer progressing after docetaxel treatment: a randomised open-label trial. Lancet. 2010;376:1147-54

10. Petrylak DP, Tangen CM, Hussain MH, et al. Docetaxel and estramustine compared with mitoxantrone and prednisone for advanced refractory prostate cancer. N Engl J Med. 2004;351:1513-20.

11. Parker C, Sartor O. Radium-223 in prostate cancer. N Engl J Med. 2013;369:1659-60.

12. Eiber M, Maurer T, Souvatzoglou M, et al. Evaluation of hybrid (6)(8)Ga-PSMA ligand PET/CT in 248 patients with biochemical recurrence after radical prostatectomy. J Nucl Med. 2015;56:668-74.

13. Braat A, Ahmadzadehfar H. Lutetium-177 labelled PSMA ligands for the treatment of metastatic castrate-resistant prostate cancer. Tijdschr Nucl Geneesk. 2016;38:1627-34.

14. Ahmadzadehfar $H$, Rahbar K, Kurpig S, et al. Early side effects and first results of radioligand therapy with (177)Lu-DKFZ-617 PSMA of castrate-resistant metastatic prostate cancer: a two-centre study. EJNMMI Res. 2015;5:114

15. Kabasakal L, AbuQbeitah M, Aygun A, et al. Pre-therapeutic dosimetry of normal organs and tissues of (177)Lu-PSMA-617 prostate-specific membrane antigen (PSMA) inhibitor in patients with castration-resistant prostate cancer. Eur J Nucl Med Mol Imaging. 2015;42:1976-83.

16. Rahbar K, Ahmadzadehfar H, Kratochwil C, et al. German multicenter study investigating 177Lu-PSMA-617 Radioligand therapy in advanced prostate cancer patients. J Nucl Med. 2017:58:85-90.

17. Ahmadzadehfar H, Aryana K, Pirayesh E, et al. The Iranian Society of Nuclear Medicine practical guideline on radioligand therapy in metastatic castrationresistant prostate cancer using 177Lu-PSMA. Iran J Nucl Med. 2018;26:2-8.

18. Heidenreich A, Bastian PJ, Bellmunt J, et al. EAU guidelines on prostate cancer. Part II: treatment of advanced, relapsing, and castration-resistant prostate cancer. Eur Urol. 2014;65:467-79.

19. Heidenreich A, Porres D. Prostate cancer: treatment sequencing for CRPC-what do we know? Nat Rev Urol. 2014;11:189-90.

20. Bostwick DG, Pacelli A, Blute M, Roche P, Murphy GP. Prostate specific membrane antigen expression in prostatic intraepithelial neoplasia and adenocarcinoma: a study of 184 cases. Cancer. 1998:82:2256-61.

21. Silver DA, Pellicer I, Fair WR, Heston WD, Cordon-Cardo C. Prostate-specific membrane antigen expression in normal and malignant human tissues. Clin Cancer Res. 1997;3:81-5

22. Wright GL Jr, Haley C, Beckett ML, Schellhammer PF. Expression of prostatespecific membrane antigen in normal, benign, and malignant prostate tissues. Urol Oncol. 1995:1:18-28.

23. Fendler WP, Kratochwil C, Ahmadzadehfar H, et al. 177Lu-PSMA-617 therapy, dosimetry and follow-up in patients with metastatic castration-resistant prostate cancer. Nuklearmedizin. 2016;55:123-8.

24. Rahbar K, Bogemann M, Ahmadzadehfar H. 177Lu-PSMA-617 radioligand therapy of mCRPC: evaluation criteria of response. Eur J Nucl Med Mo Imaging. 2017;44:166-7.

25. Rahbar K, Schmidt M, Heinzel A, et al. Response and tolerability of a single dose of 177Lu-PSMA-617 in patients with metastatic castration-resistant prostate Cancer: a multicenter retrospective analysis. J Nucl Med. 2016:57:1334-8.

26. Ahmadzadehfar $H$, Eppard E, Kurpig S, et al. Therapeutic response and side effects of repeated radioligand therapy with 177Lu-PSMA-DKFZ-617 of castrate-resistant metastatic prostate cancer. Oncotarget. 2016;7:12477-88.

27. Ahmadzadehfar $\mathrm{H}$, Wegen S, Yordanova A, et al. Overall survival and response pattern of castration-resistant metastatic prostate cancer to multiple cycles of radioligand therapy using [177Lu]Lu-PSMA-617. Eur J Nuc Med Mol Imaging. 2017:44:1448-54 
28. Ahmadzadehfar H, Zimbelmann S, Yordanova A, et al. Radioligand therapy of metastatic prostate cancer using 177Lu-PSMA-617 after radiation exposure to 223Ra-dichloride. Oncotarget. 2017:8:55567-74.

29. Ferdinandus J, Eppard E, Gaertner FC, et al. Predictors of response to Radioligand therapy of metastatic castrate-resistant prostate Cancer with 177Lu-PSMA-617. J Nucl Med. 2017:58:312-9.

30. Kulkarni HR, Singh A, Schuchardt C, et al. PSMA-based Radioligand therapy for metastatic castration-resistant prostate Cancer: the Bad Berka experience since 2013. J Nucl Med. 2016;57:97S-104S

31. Rathke H, Giesel FL, Flechsig P, et al. Repeated (177)Lu-labeled PSMA-617 Radioligand therapy using treatment activities of up to $9.3 \mathrm{GBq}$. J Nucl Med. 2018;59:459-65.

32. Kratochwil C, Giesel FL, Stefanova M, et al. PSMA-targeted radionuclide therapy of metastatic castration-resistant prostate Cancer with 177Lu-labeled PSMA-617. J Nucl Med. 2016:57:1170-6.

33. Fendler WP, Reinhardt $\mathrm{S}$, Ilhan $\mathrm{H}$, et al. Preliminary experience with dosimetry, response and patient reported outcome after 177Lu-PSMA-617 therapy for metastatic castration-resistant prostate cancer. Oncotarget. 2017;8:3581-90.

34. Heck MM, Retz M, D'Alessandria C, et al. Systemic Radioligand therapy with (177)Lu labeled prostate specific membrane antigen ligand for imaging and therapy in patients with metastatic castration resistant prostate Cancer. J Urol. 2016;196:382-91.

35. Kratochwil C, Bruchertseifer F, Rathke H, et al. Targeted alpha-therapy of metastatic castration-resistant prostate Cancer with 225Ac-PSMA-617: dosimetry estimate and empiric dose finding. J Nucl Med. 2017;58:1624-31.

36. Kratochwil C, Bruchertseifer F, Giesel FL, et al. 225Ac-PSMA-617 for PSMAtargeted alpha-radiation therapy of metastatic castration-resistant prostate Cancer. J Nucl Med. 2016;57:1941-4.

37. Zechmann CM, Afshar-Oromieh A, Armor T, et al. Radiation dosimetry and first therapy results with a (124)I/ (131)l-labeled small molecule (MIP-1095) targeting PSMA for prostate cancer therapy. Eur J Nucl Med Mol Imaging. 2014;41:1280-92.

38. Afshar-Oromieh A, Haberkorn U, Zechmann C, et al. Repeated PSMA-targeting radioligand therapy of metastatic prostate cancer with 131I-MIP-1095. Eur J Nucl Med Mol Imaging. 2017:44:950-9.

39. Rahbar K, Bode A, Weckesser M, et al. Radioligand therapy with 177Lu-PSMA-617 as a novel therapeutic option in patients with metastatic castration resistant prostate Cancer. Clin Nucl Med. 2016;41:522-8.

40. Ahmadzadehfar $\mathrm{H}$, Schlolaut S, Fimmers $\mathrm{R}$, et al. Predictors of overall survival in metastatic castration-resistant prostate cancer patients receiving [177Lu]Lu-PSMA-617 radioligand therapy. Oncotarget. 2017;8(61):103108-16.

41. Rahbar K, Boegemann M, Yordanova A, et al. PSMA targeted radioligandtherapy in metastatic castration resistant prostate cancer after chemotherapy, abiraterone and/or enzalutamide. A retrospective analysis of overall survival. Eur J Nucl Med Mol Imaging. 2018;45(1):12-19.

42. Gourni E, Henriksen G. Metal-based PSMA Radioligands. Molecules. 2017; 22(4)

43. Delker A, Fendler WP, Kratochwil C, et al. Dosimetry for (177)Lu-DKFZ-PSMA-617: a new radiopharmaceutical for the treatment of metastatic prostate cancer. Eur J Nucl Med Mol Imaging. 2016:43:42-51.

44. Guerriero F, Ferrari ME, Botta F, et al. Kidney dosimetry in (1)(7)(7)Lu and (9)(0)Y peptide receptor radionuclide therapy: influence of image timing, time-activity integration method, and risk factors. Biomed Res Int. 2013;2013:935351.

45. Gaertner FC, Halabi K, Ahmadzadehfar H, et al. Uptake of PSMA-ligands in normal tissues is dependent on tumor load in patients with prostate cancer. Oncotarget. 2017;8:55094-103.

46. Vallabhajosula S, Goldsmith SJ, Hamacher KA, et al. Prediction of myelotoxicity based on bone marrow radiation-absorbed dose: radioimmunotherapy studies using 90Y- and 177Lu-labeled J591 antibodies specific for prostate-specific membrane antigen. J Nucl Med. 2005;46:850-8.

47. Yordanova A, Becker A, Eppard E, et al. The impact of repeated cycles of radioligand therapy using [177Lu]Lu-PSMA-617 on renal function in patients with hormone refractory metastatic prostate cancer. Eur J Nucl Med Mol Imaging. 2017:44:1473-9.

48. Heck MM, Retz M, D'Alessandria C, et al. Systemic radioligand therapy with Lu-PSMA-I\&T in patients with metastatic castration-resistant prostate cancer. J Urol. 2016;196(2):382-91
49. Miederer $M$, Henriksen $G$, Alke A, et al. Preclinical evaluation of the alpha-particle generator nuclide 225Ac for somatostatin receptor radiotherapy of neuroendocrine tumors. Clin Cancer Res. 2008;14:3555-61.

50. Kratochwil C, Bruchertseifer F, Rathke H, et al. Targeted alpha therapy of mCRPC with (225)actinium-PSMA-617: swimmer-plot analysis suggests efficacy regarding duration of tumor-control. J Nucl Med. 2018;59(5):795-802.

51. Baum RP, Kulkarni HR, Schuchardt C, Singh A, Wirtz M, Wiessalla S, Schottelius M, Mueller D, Klette I, Wester HJ. Lu-Labe led Prostate-Specific Membrane Antigen Radioligand Therapy of Metastatic Castration-Resistant Prostate Cancer: Safety and Efficacy. J Nucl Med. 2016;57(7):1006-13.

52. Yadav MP, Ballal S, Tripathi M, Damle NA, Sahoo RK, Seth A, Bal C. Lu-DKFZPSMA-617 therapy in metastatic castration resistant prostate cancer: safety, efficacy, and quality of life assessment. Eur J Nucl Med Mol Imaging. 2017; 44(1):81-91

\section{Ready to submit your research? Choose BMC and benefit from:}

- fast, convenient online submission

- thorough peer review by experienced researchers in your field

- rapid publication on acceptance

- support for research data, including large and complex data types

- gold Open Access which fosters wider collaboration and increased citations

- maximum visibility for your research: over $100 \mathrm{M}$ website views per year

At BMC, research is always in progress.

Learn more biomedcentral.com/submissions 